\title{
Neonatal jaundice and risks of childhood allergic diseases: a population-based cohort study
}

\author{
Chang-Ching Wei ${ }^{1,2}$, Cheng-Li Lin ${ }^{2,3}$, Te-Chun Shen ${ }^{2,4}$ and Chia-Hung Kao ${ }^{5,6}$
}

BACKGROUND: Only a few studies have systemically analyzed the association between neonatal jaundice and childhood-onset allergic diseases.

METHODS: From 2000 to 2007, 27,693 neonates with newly diagnosed neonatal jaundice and 55,367 matched nonneonatal jaundice cohorts were identified. The incidences and hazard ratios (HRs) of five allergic diseases, namely allergic conjunctivitis (AC), allergic rhinitis (AR), atopic dermatitis (AD), asthma, and urticaria, by the end of 2008 were calculated.

RESULTS: The incidence density and HRs of the five allergic diseases were greater in the neonatal jaundice cohort than in the nonneonatal jaundice cohort, and the HRs declined modestly with age. The HRs for AR ( $H R=2.51,95 \%$ confidence interval $(\mathrm{Cl})=2.43-2.59)$ and $\mathrm{AD}(\mathrm{HR}=2.51,95 \% \mathrm{Cl}=2.40-2.62)$ were the highest, and that for urticaria was the lowest ( $H R=$ $2.06,95 \% \mathrm{Cl}=1.94-2.19)$. The HRs of allergic diseases were substantially greater for boys and those requiring phototherapy. The HRs of the allergic diseases, except urticaria ( $H R=2.49,95 \%$ $\mathrm{Cl}=1.57-3.97)$, were not significantly different between the neonatal jaundice regardless of whether the patients received exchange transfusion.

CONCLUSION: Neonatal jaundice is associated with the development of allergic diseases in early childhood.

N eonatal jaundice results from a predisposition to the production of bilirubin in newborn infants, especially preterm infants, and their limited ability to excrete it. Infants have higher rates of bilirubin production because they have red blood cells with a higher turnover and a shorter life span, and they have a limited ability to excrete it because of the reduced activity of glucuronyl transferase, which converts bilirubin into water-soluble bilirubin glucuronides $(1,2)$. When the concentration of serum bilirubin exceeds its protein binding capacity, bilirubin crystals aggregate and precipitate in neurons $(1,2)$. In the past decades, phototherapy and exchange transfusion have been widely used to prevent hyperbilirubinemia-induced neurological damage $(3,4)$. Bilirubin, a powerful antioxidant, may be distributed to other tissues (5-7). In addition, bilirubin has been found to have immunomodulatory effects, inhibiting the T helper-1 (Th1) cell response and interleukin-2 (IL-2) production (8). A reduction in the level of IL-2 skews the Th1/ Th2 balance toward Th2, thereby increasing the risk of atopy development $(9,10)$. Although previous studies have reported the relationship of neonatal jaundice with childhood asthma, few studies have systemically analyzed the association between neonatal jaundice and common childhood allergic diseases (11-15). The prevalence of allergic diseases has risen dramatically worldwide, creating a health care burden. Identifying the pathologic agents and risk factors of allergic diseases may facilitate disease prevention and more specific treatment. Therefore, there is great interest in studying the long-term effects of bilirubin on the development of atopic diathesis. Allergic conjunctivitis (AC), allergic rhinitis (AR), atopic dermatitis (AD), asthma, and urticaria are common organ-specific childhood allergic diseases (10). Hence, we conducted a nationwide birth cohort study to systemically investigate the incidence and relative risk of common allergic diseases in children with neonatal jaundice. We hypothesized that bilirubin would increase the future risk of atopic diathesis.

\section{RESULTS}

The baseline demographic and clinical characteristics of the sample are presented in Table 1. Among the two cohorts, of which one comprised children with neonatal jaundice and one comprised nonneonatal jaundice controls, $60.2 \%$ were boys. The neonatal jaundice cohort was significantly more likely than the nonneonatal jaundice cohort to have fetal and newborn respiratory conditions (6.3 vs. $1.6 \%$ ), infections (14.6 vs. $2.0 \%)$, preterm delivery and low birth weight (9.3 vs. $1.7 \%$ ), other birth conditions (8.6 vs. 1.7\%), and glucose-6-phosphate dehydrogenase deficiency (1.2 vs. $0.1 \%$ ). These perinatal conditions were confounding factors and were adjusted later to study the association between allergic diseases and neonatal jaundice.

In all age groups, the incidences of all allergic diseases were higher for the neonatal jaundice cohort than for the nonneonatal jaundice cohort (Table 2). The HRs for AR

'Children's Hospital, China Medical University Hospital, Taichung, Taiwan; ${ }^{2}$ College of Medicine, China Medical University, Taichung, Taiwan; ${ }^{3}$ Management Office for Health Data, China Medical University Hospital, Taichung, Taiwan; ${ }^{4}$ Division of Pulmonary and Critical Care Medicine, Department of Internal Medicine, Taichung, Taiwan; ${ }^{5}$ Graduate Institute of Clinical Medicine Science and School of Medicine, College of Medicine, China Medical University, Taichung, Taiwan; ${ }^{6}$ Department of Nuclear Medicine and PET Center, China Medical University Hospital, Taichung, Taiwan. Correspondence: Chia-Hung Kao (d10040@mail.cmuh.org.tw) 
Table 1. Comparison of baseline demographics between birth cohorts with and without neonatal jaundice in Taiwan in 2000-2007 followed until 2008

\begin{tabular}{|c|c|c|c|c|}
\hline & \multicolumn{4}{|c|}{ Neonatal jaundice } \\
\hline & \multicolumn{2}{|c|}{ No $(N=55,367)$} & \multicolumn{2}{|c|}{ Yes $(N=27,693)$} \\
\hline & $N$ & $\%$ & $n$ & $\%$ \\
\hline Age, days, mean (SD) a & 15 & 9 & 6 & 6 \\
\hline \multicolumn{5}{|l|}{ Sex } \\
\hline Girl & 22,051 & 40 & 11,028 & 40 \\
\hline Boy & 33,316 & 60 & 16,665 & 60 \\
\hline \multicolumn{5}{|l|}{ Urbanization $^{\mathrm{b}}$} \\
\hline 1 (highest) & 15,918 & 29 & 7,959 & 29 \\
\hline 2 & 17,246 & 31 & 8,623 & 31 \\
\hline 3 & 8,901 & 16 & 4,453 & 16 \\
\hline 4 (lowest) & 13,302 & 24 & 6,658 & 24 \\
\hline \multicolumn{5}{|l|}{ Parental occupation } \\
\hline White collar & 32,986 & 60 & 16,498 & 60 \\
\hline Blue collar & 12,813 & 23 & 6,409 & 23 \\
\hline Others ${ }^{c}$ & 9,568 & 17 & 4,786 & 17 \\
\hline \multicolumn{5}{|l|}{ Comorbidity } \\
\hline $\begin{array}{l}\text { Fetal and newborn } \\
\text { respiratory conditions* }\end{array}$ & 860 & 1.6 & 1,751 & 6.3 \\
\hline Infections* & 1,106 & 2.0 & 4,033 & 14.6 \\
\hline $\begin{array}{l}\text { Preterm/low birth } \\
\text { weight* }\end{array}$ & 944 & 1.7 & 2,583 & 9.3 \\
\hline Birth weight $<1,000 \mathrm{~g}$ & 84 & 0.2 & 47 & 0.2 \\
\hline Birth weight $1,000-2,499 \mathrm{~g}$ & 739 & 1.3 & 2,330 & 8.4 \\
\hline Other birth conditions* & 927 & 1.7 & 2,369 & 8.6 \\
\hline G-6-PDd deficiency* & 36 & 0.1 & 330 & 1.2 \\
\hline
\end{tabular}

$\chi^{2}$ test, and ${ }^{a}$-test comparing subjects with neonatal jaundice and without neonatal jaundice. ${ }^{\text {TThe }}$ urbanization level was categorized by the population density of the residential area. 'Other occupations included primarily retired, unemployed, or low income populations. ${ }^{d}$ G-6-PD: glucose-6-phosphate dehydrogenase. ${ }^{*} P$ value $<0.0001$.

$(\mathrm{HR}=2.51,95 \%$ confidence interval $(\mathrm{CI})=2.43-2.59)$ and $\mathrm{AD}(\mathrm{HR}=2.51,95 \% \mathrm{CI}=2.40-2.62)$ were the highest, and that for urticaria was the lowest $(\mathrm{HR}=2.06,95 \% \mathrm{CI}=1.94-$ 2.19). Differences in the disease type-specific incidence were greatest for AR (68 per 1,000 person-years for the neonatal jaundice cohort vs. 26 per 1,000 person-years for the nonneonatal jaundice cohort), whereas the smallest difference was noted for urticaria (13 per 1,000 person-years for the neonatal jaundice cohort vs. 6 per 1,000 person-years for the nonneonatal jaundice cohort). The incidences of all allergic diseases and sex-specific adjusted HRs were higher in boys in both cohorts.

The Kaplan-Meier plots in Figure 1 show the 9-y probabilities of developing $\mathrm{AC}, \mathrm{AR}, \mathrm{AD}$, asthma, and urticaria. The cumulative incidence of each allergic disease was higher for the neonatal jaundice cohort than for the nonneonatal jaundice cohort (log-rank test, $P<0.001$ ). The difference in cumulative incidences between the two cohorts was the greatest for $\mathrm{AR}$ and the smallest for urticaria.
Phototherapy was significantly associated with a higher risk of allergic diseases (Table 3), and exchange transfusion was significantly associated with a higher risk of urticaria (adjusted HR $=2.52,95 \% \mathrm{CI}=1.59-4.02$ ) (Table 4 ). By 6 y of age, the children in both cohorts had higher incidence rates for all allergic diseases, and the children in the neonatal jaundice cohort had higher adjusted HRs for most of the allergic diseases except for AR (Table 5). We further investigated the incidence rates and hazard ratios (HRs) of allergic diseases in children with or without neonatal jaundice stratified by full term, birth weight $<1,000 \mathrm{~g}$, and birth weight $1,000-2,499 \mathrm{~g}$. Increased HRs for the five allergic diseases were consistently found in the three stratified groups (Table 6).

\section{DISCUSSION}

This population-based cohort study investigated the incidences and relative risks of five allergic diseases in children with neonatal jaundice in comparison with those without neonatal jaundice. The results consistently show higher risks of allergic diseases in childhood among those with neonatal jaundice. Furthermore, these risks were associated with a high bilirubin level requiring phototherapy.

Four previous studies have reported the association between neonatal jaundice and asthma (11-14). Two studies in Sweden conducted by Aspberg et al. first reported the association of neonatal jaundice and phototherapy with childhood asthma requiring hospitalization (odds ratio $=1.27,95 \% \mathrm{CI}=1.08,1.50$ ) and confirmed that neonatal jaundice and phototherapy are associated with the use of antiasthmatic drugs before $12 \mathrm{y}$ of age (odds ratio $=1.30,95 \% \mathrm{CI}=1.16,1.47)$ in their subsequent analysis $(11,12)$. In another birth cohort study performed in the United States during 1959-1965, when phototherapy was unavailable, neonatal hyperbilirubinemia was associated with an increased risk of childhood asthma (odds ratio $=1.61,95 \% \mathrm{CI}=1.04$, 2.08) (14). In the current study, the HRs of the five allergic diseases were consistently higher in the neonatal jaundice cohort than in the nonneonatal jaundice cohort when they were stratified by full term and low birth weight. A strong association between preterm delivery and the diagnosis of asthma has been reported $(16,17)$. In the current study, we found that the incidence rates of asthma were the highest in children with a birth weight $<1,000 \mathrm{~g}$ in both cohorts. However, the HRs of asthma were the lowest in the children with a birth weight $<1,000 \mathrm{~g}$. These findings are in line with those of previous studies. Two recent Taiwanese studies showed an association between neonatal jaundice and asthma (13) (odds ratio $=1.64,95 \% \mathrm{CI}=$ $1.36,1.98$ ) as well as $\mathrm{AR}(15)$ (odds ratio $=1.46,95 \% \mathrm{CI}=1.24$, 1.72). However, these two studies used the claims data of a sample of one million patients from the National Health Insurance Research Database (NHIRD), thus limiting the number of study patients and resulting in a loss of statistical significance for other atopic diseases. Moreover, no previous studies have analyzed as many allergic diseases as the current study did. This study is the first to report the risks of each allergic disease (AC, $\mathrm{AR}, \mathrm{AD}$, asthma, and urticaria) in children with neonatal jaundice and that phototherapy was associated with increased risks 
Table 2. Comparison of incidence density of allergic diseases between birth cohorts with neonatal jaundice $(N=27,693)$ or without neonatal jaundice $(N=55,367)$ by sex in Taiwan in $2000-2007$ followed until 2008

\begin{tabular}{|c|c|c|c|c|c|c|c|c|}
\hline \multirow[b]{2}{*}{ Allergic disease/sex } & \multicolumn{3}{|c|}{ Non-neonatal jaundice } & \multicolumn{3}{|c|}{ Neonatal jaundice } & \multirow[b]{2}{*}{$\mathrm{cHR}(95 \% \mathrm{Cl})$} & \multirow[b]{2}{*}{$\mathrm{aHR}(95 \% \mathrm{Cl})$} \\
\hline & Event & PY & IR & Event & PY & IR & & \\
\hline Allergic conjunctivitis & 4,058 & 327,043 & 12 & 4,315 & 154,835 & 28 & $2.24(2.15,2.34)^{*}$ & $2.20(2.09,2.32)^{*}$ \\
\hline Boy & 2,591 & 196,043 & 13 & 2,803 & 92,203 & 30 & $2.29(2.18,2.42)^{*}$ & $2.28(2.14,2.43)^{* *}$ \\
\hline Allergic rhinitis & 8,144 & 309,661 & 26 & 9,055 & 134,172 & 68 & $2.51(2.43,2.59)^{*}$ & $2.52(2.43,2.61)^{*}$ \\
\hline Boy & 5,265 & 184,658 & 29 & 5,786 & 79,177 & 73 & $2.50(2.41,2.60)^{*}$ & $2.51(2.40,2.62)^{* *}$ \\
\hline Asthma & 5,644 & 320,113 & 18 & 6,309 & 146,243 & 43 & $2.41(2.33,2.50)^{*}$ & $2.35(2.25,2.45)^{*}$ \\
\hline Girl & 1,950 & 129,057 & 15 & 2,211 & 59,699 & 37 & $2.43(2.28,2.58)^{*}$ & $2.33(2.16,2.50)^{* *}$ \\
\hline Boy & 3,694 & 191,056 & 19 & 4,098 & 86,543 & 47 & $2.41(2.30,2.52)^{*}$ & $2.37(2.24,2.49)^{* *}$ \\
\hline Atopic dermatitis & 3,735 & 32,359 & 12 & 4,430 & 148,264 & 30 & $2.51(2.40,2.62)^{*}$ & $2.49(2.37,2.62)^{*}$ \\
\hline Girl & 776 & 133,091 & 6 & 817 & 64,606 & 13 & $2.15(1.95,2.37)^{*}$ & $1.97(1.75,2.21)^{* *}$ \\
\hline Boy & 1,290 & 200,170 & 6 & 1,272 & 97,488 & 13 & $2.01(1.86,2.17)^{*}$ & $1.94(1.77,2.13)^{* *}$ \\
\hline
\end{tabular}

$H R$, hazard ratio; IR, incidence rate, per 1,000 person-years; $\mathrm{PY}$, person-years.

Crude $H R^{a}$, relative hazard ratio. Adjusted $H R^{b}$, adjusted for age, sex and comorbidities of fetal and newborn respiratory conditions, infections, preterm, low birth weight, other birth conditions, and Glucose-6-phosphate dehydrogenase (G6PD) deficiency. ${ }^{*} P<0.05,{ }^{* *} P<0.001$.
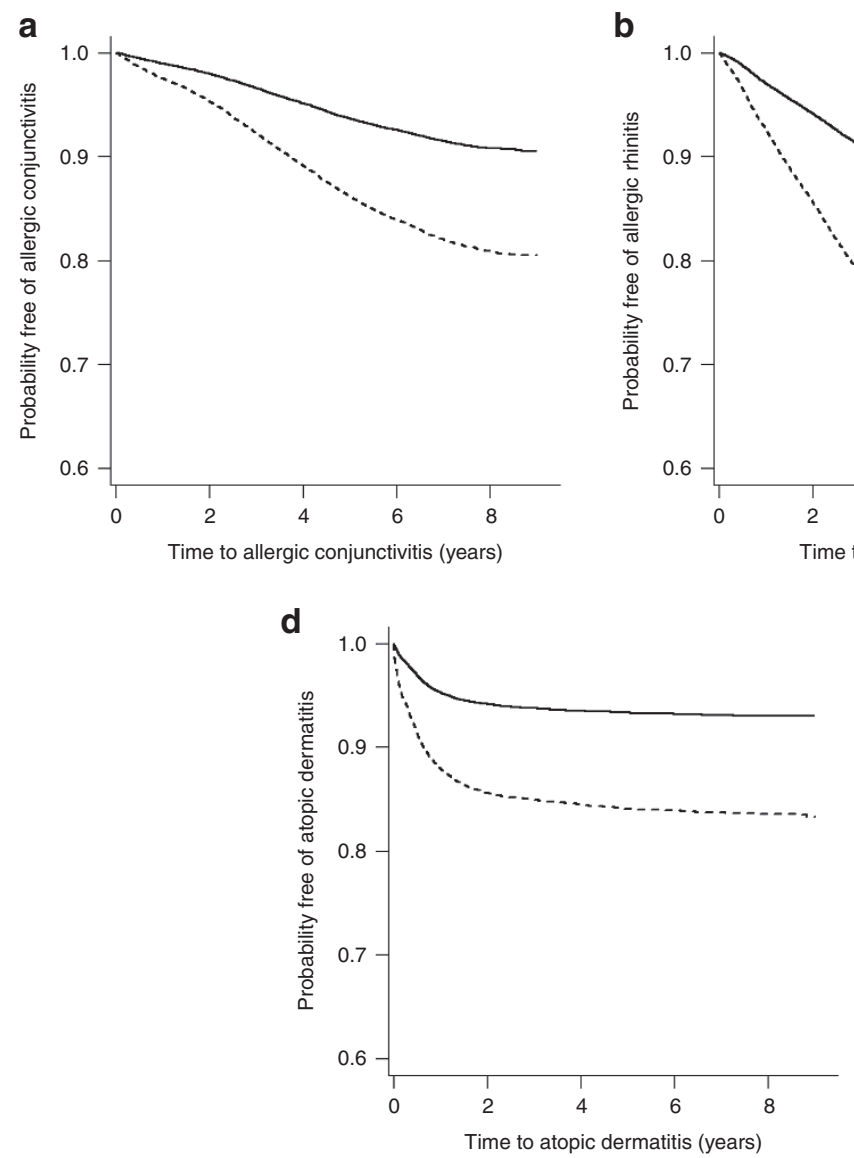
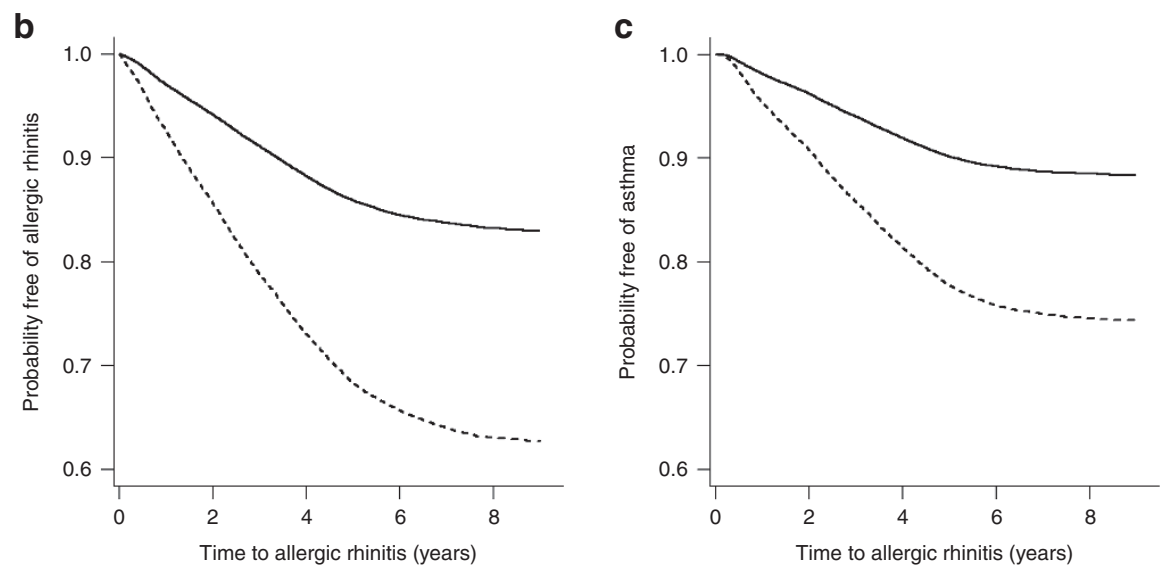

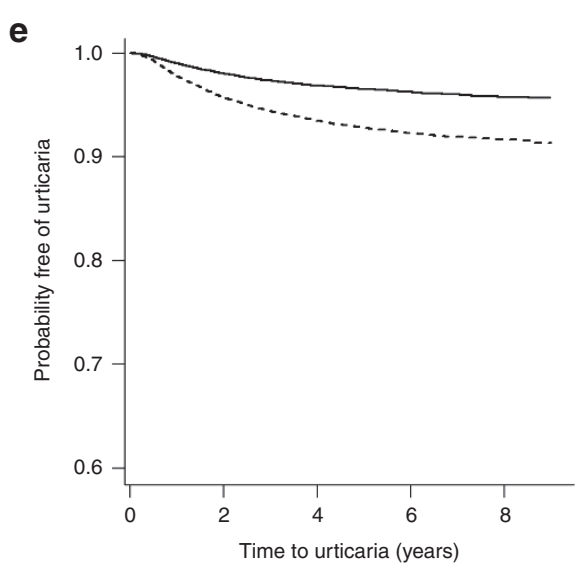

Figure 1. Probability of being free of allergic conjunctivitis (a), allergic rhinitis (b), asthma (c), atopic dermatitis (d), and urticaria (e) for patients with (dashed line) and without (solid line) neonatal jaundice. 


\section{Articles | Wei et al.}

Table 3. Comparison of incidence density of allergic diseases between neonatal jaundice cohort $(N=27,693)$ with phototherapy and without phototherapy in Taiwan in 2000-2007 followed until 2008

\begin{tabular}{|c|c|c|c|c|c|c|c|c|}
\hline \multirow[b]{2}{*}{ Allergic disease } & \multicolumn{3}{|c|}{ No Phototherapy } & \multicolumn{3}{|c|}{ Phototherapy } & \multirow[b]{2}{*}{$\mathrm{cHR}(95 \% \mathrm{Cl})$} & \multirow[b]{2}{*}{$\operatorname{aHR}(95 \% \mathrm{Cl})$} \\
\hline & Event & PY & IR & Event & PY & IR & & \\
\hline Allergic conjunctivitis & 1,830 & 76,920 & 24 & 2,485 & 77,914 & 32 & $1.33(1.25,1.41)^{* *}$ & $1.38(1.30,1.47)^{* *}$ \\
\hline Asthma & 3,995 & 67,813 & 59 & 5,060 & 66,359 & 76 & $1.17(1.11,1.22)^{* *}$ & $1.21(1.15,1.27)^{* *}$ \\
\hline Atopic dermatitis & 2,095 & 73,527 & 29 & 2,335 & 74,737 & 31 & $1.13(1.07,1.20)^{* *}$ & $1.22(1.14,1.29)^{* *}$ \\
\hline
\end{tabular}

$H R$, hazard ratio; IR, incidence rate, per 1,000 person-years; $P Y$, person-years.

Crude HRa, relative hazard ratio. Adjusted HR' adjusted for age, sex and comorbidities of fetal and newborn respiratory conditions, infections, preterm, low birth weight, other birth conditions, and Glucose-6-phosphate dehydrogenase (G6PD) deficiency. ${ }^{*} P<0.05,{ }^{* *} P<0.001$.

Table 4. Comparison of incidence density of allergic diseases between neonatal jaundice cohort $(N=27,693)$ with exchange transfusion and without exchange transfusion in Taiwan in 2000-2007 followed until 2008

\begin{tabular}{|c|c|c|c|c|c|c|c|c|}
\hline \multirow[b]{2}{*}{ Allergic disease } & \multicolumn{3}{|c|}{ No Exchange transfusion } & \multicolumn{3}{|c|}{ Exchange transfusion } & \multirow[b]{2}{*}{$\mathrm{cHR}(95 \% \mathrm{Cl})$} & \multirow[b]{2}{*}{$\operatorname{aHR}(95 \% \mathrm{Cl})$} \\
\hline & Event & PY & IR & Event & PY & $\mathrm{IR}$ & & \\
\hline Allergic conjunctivitis & 4,297 & 154,254 & 28 & 18 & 581 & 31 & $1.12(0.71,1.78)$ & $1.14(0.72,1.81)$ \\
\hline Asthma & 6,288 & 145,691 & 43 & 21 & 552 & 38 & $0.92(0.60,1.41)$ & $0.91(0.60,1.40)$ \\
\hline Atopic dermatitis & 4,414 & 147,716 & 30 & 16 & 548 & 29 & $1.04(0.64,1.70)$ & $1.06(0.65,1.74)$ \\
\hline
\end{tabular}

$H R$, hazard ratio; IR, incidence rate, per 1000 person-years; $P Y$, person-years.

Crude HRa, relative hazard ratio. Adjusted $\mathrm{HR}^{\mathrm{b}}$, adjusted for age, sex and comorbidities of fetal and newborn respiratory conditions, infections, preterm, low birth weight, other birth conditions, and Glucose-6-phosphate dehydrogenase (G6PD) deficiency. ${ }^{*} P<0.001$.

Table 5. Comparison of incidence density of allergic diseases between birth cohorts with neonatal jaundice $(N=27,693)$ or without neonatal jaundice $(N=55,367)$ by follow-up years in Taiwan in 2000-2007 followed until 2008

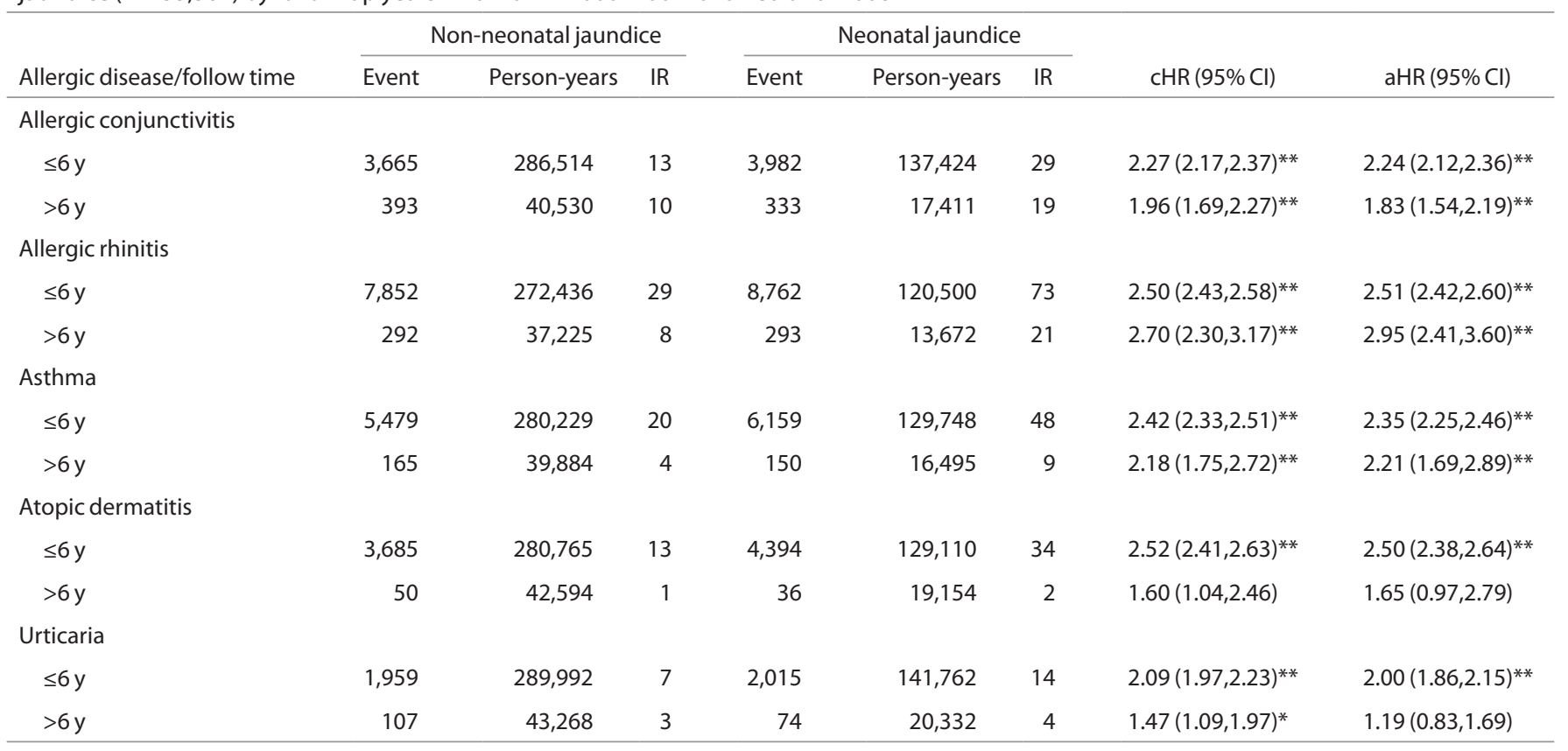

$\mathbb{R}$, incidence rate, per 1,000 person-years.

Crude HRa, relative hazard ratio. Adjusted $H R^{b}$, adjusted for age, sex, and comorbidities of fetal and newborn respiratory conditions, infections, preterm, low birth weight, other birth conditions, and Glucose-6-phosphate dehydrogenase (G6PD) deficiency. Follow time was divided into before school-age $(\leq 6 \mathrm{y})$ and $s c h o o l$ age $(>6 \mathrm{y})$. ${ }^{*} P<0.05,{ }^{* *} P<0.001$.

of these diseases. These associations were consistent among all five diseases regardless of age and sex. The incidence rate for AR was the highest, with an overall incidence density rate of 68 per
1,000 person-years in the neonatal jaundice cohort, representing a 2.4-fold increase over the incidence rate for AC and a 5.2fold increase over the incidence rate for urticaria. 
Table 6. Comparison of incidence density of allergic diseases between birth cohorts with neonatal jaundice $(N=27,693)$ or without neonatal jaundice $(N=55,367)$ by birth weight in Taiwan in 2000-2007 followed until 2008

\begin{tabular}{|c|c|c|c|c|c|c|c|c|}
\hline \multirow[b]{2}{*}{ Allergic disease/birth weight } & \multicolumn{3}{|c|}{ Non-neonatal jaundice } & \multicolumn{3}{|c|}{ Neonatal jaundice } & \multirow[b]{2}{*}{$\mathrm{cHR}(95 \% \mathrm{Cl})$} & \multirow[b]{2}{*}{$\operatorname{aHR}(95 \% \mathrm{Cl})$} \\
\hline & Event & PY & IR & Event & PY & IR & & \\
\hline \multicolumn{9}{|l|}{ Allergic conjunctivitis } \\
\hline$<1,000 \mathrm{~g}$ & 6 & 366 & 16 & 10 & 206 & 49 & $2.78(1.01,7.67)^{*}$ & $2.43(0.68,8.69)$ \\
\hline $1,000-2,499 \mathrm{~g}$ & 67 & 4,324 & 16 & 410 & 12,825 & 32 & $2.05(1.59,2.66)^{* * *}$ & $2.22(1.62,3.04)^{* * *}$ \\
\hline Full term & 7,979 & 304,523 & 26 & 8,289 & 121,195 & 68 & $2.55(2.47,2.63)^{* * *}$ & $2.55(2.46,2.64)^{* * * *}$ \\
\hline$<1,000 \mathrm{~g}$ & 17 & 335 & 51 & 7 & 221 & 32 & $0.63(0.26,1.51)$ & $0.45(0.16,1.26)$ \\
\hline $1,000-2,499 \mathrm{~g}$ & 126 & 4,031 & 31 & 694 & 11,661 & 60 & $1.87(1.54,2.26)^{* * *}$ & $2.08(1.64,2.64)^{* * * *}$ \\
\hline \multicolumn{9}{|l|}{ Asthma } \\
\hline Full term & 5,527 & 314,764 & 18 & 5,688 & 132,720 & 43 & $2.41(2.32,2.50)^{* * *}$ & $2.36(2.26,2.47)^{* * * *}$ \\
\hline Full term & 3,680 & 317,798 & 12 & 4,055 & 134,109 & 30 & $2.53(2.42,2.64)^{* * *}$ & $2.49(2.36,2.63)^{* * * *}$ \\
\hline$<1,000 \mathrm{~g}$ & 4 & 371 & 11 & 5 & 229 & 22 & $1.87(0.50,3.97)$ & $1.34(0.24,7.60)$ \\
\hline $1,000-2,499 \mathrm{~g}$ & 42 & 4,382 & 10 & 338 & 12,740 & 27 & $2.61(1.89,3.59)^{* * *}$ & $2.74(1.87,4.02)^{* * *}$ \\
\hline \multicolumn{9}{|l|}{ Urticaria } \\
\hline Full term & 2,031 & 327,545 & 6 & 1,908 & 146,808 & 13 & $2.08(1.95,2.21)^{* * *}$ & $1.97(1.83,2.12)^{* * * *}$ \\
\hline$<1,000 \mathrm{~g}$ & 4 & 375 & 11 & 2 & 248 & 8 & $0.76(0.14,4.17)$ & $2.35(0.21,26.0)$ \\
\hline $1,000-2,499 \mathrm{~g}$ & 28 & 4,482 & 6 & 168 & 13,749 & 12 & $1.91(1.28,2.85)^{* *}$ & $1.20(0.73,1.95)$ \\
\hline
\end{tabular}

$H R$, hazard ratio; $I R$, incidence rate, per 1,000 person-years; $P Y$, person-years.

Crude HRa, relative hazard ratio. Adjusted HR' adjusted for age, sex, and comorbidities of fetal and newborn respiratory conditions, infections, other birth conditions, and

Glucose-6-phosphate dehydrogenase (G6PD) deficiency. ${ }^{*} P<0.05,{ }^{* *} P<0.01,{ }^{* *} P<0.001$.

Sex-specific relative hazards were higher among boys for all allergic diseases, indicating that the impact of neonatal jaundice is greater among boys than among girls. The age-specific relative hazards were higher in children $\leq 6 \mathrm{y}$, indicating that neonatal jaundice is associated with the development of allergic diseases since early childhood. The discrepancy between sex and age groups has not been well documented in previous studies.

With the increasing prevalence of allergic diseases worldwide, much interest has been focused on studying the effects of environmental stimuli on the early development of the immune system. Being a noninvasive method and as effective as exchange transfusion in preventing bilirubin encephalopathy, phototherapy had been widely applied to treat neonatal jaundice for many decades. However, recent evidence has revealed that phototherapy may affect the Th2/Th1 switch, which contributes to the development of allergic diseases. First, phototherapy may alter the Th2/Th1 cytokine profile, increasing the levels of tumor necrosis factor- $\alpha$, IL-1- $\beta$, and IL- 8 and a reducing the level of IL-6 in newborn infants $(18,19)$. Second, phototherapy causing DNA damage to lymphocytes can affect the genes regulating the Th2/Th1 switch $(20,21)$. Third, UV light, despite being a small component of phototherapy, can activate the inflammatory pathways, leading to allergic diseases or autoimmune disorders (22). Fourth, a proper increase in the level of unconjugated bilirubin inhibits complement activation, prevents leukocyte migration, and protects infants from oxidative stress (23). This evidence supports our findings that interfering with physiological bilirubin metabolism by administering neonatal phototherapy may be a risk factor for allergic diseases in later life.

Exchange transfusion was a common procedure for treating extremely severe hyperbilirubinemia in neonates until the National Institute of Child Health and Human Development reported that phototherapy was as effective as exchange transfusion in preventing bilirubin encephalopathy in $1985(24,25)$. Since then, phototherapy has been widely adopted as the primary therapy for neonatal jaundice. Only one study has described increased levels of serum immunoglobulin E ( $\operatorname{IgE}$ ) in infants after exchange transfusion for neonatal jaundice (26). However, the long-term effects of exchange transfusion on atopy in infants with neonatal jaundice remain unclear. The results of the current study, investigating for the first time the association between exchange transfusion and a long-term risk of allergic diseases, showed that only urticaria had a significant association with exchange transfusion, while it had the weakest association with phototherapy compared with other allergic diseases. The mean time from the diagnosis of neonatal 
jaundice to urticaria is $3.39 \mathrm{y}(\mathrm{SD}=2.26)$, and hence, urticaria is not only an immediate reaction to exchange transfusion. Because only a few studies are reported in literatures and few patients required exchange transfusion in the current study, further study is warranted to elucidate the association between exchange transfusion and atopy as well as the mechanisms underlying this association.

The mechanisms underlying the effects of neonatal jaundice on allergic diseases remain unclear. Previous studies have suggested that bilirubin may inhibit the Th1 cell response, and intracellular accumulation of unconjugated bilirubin may inhibit the production of IL-2 $(8,18,19)$. A reduction in the level of IL-2 skews the Th1/Th2 balance toward Th2, thereby increasing the risk of developing allergic diseases $(9,10)$. Glutathione S-transferases are a large family of proteins that participate in the metabolism of reactive oxygen species and detoxification of xenobiotics. Polymorphisms in the genes of glutathione S-transferase indicate an association between neonatal hyperbilirubinemia and allergy, asthma, and AD (27-30). Bilirubin may increase intestinal protein permeability and may be associated with a higher risk of developing an allergy to cow milk protein in young infants (31). Our findings suggest that atopy is not a disease of a single organ, but rather a systemic response to common pathophysiology consequences. These allergic diseases may progress over time and may occur concurrently. The pathophysiologic complexity of the relationship between neonatal jaundice and allergic consequences warrants further exploration.

The strengths of this study are as follows. First, we employed a large population-based dataset to quantify the atopic risk in children with neonatal jaundice and used a retrospective cohort design, which may minimize the selection bias observed in previous case-control studies $(11,12)$. In 1995, Taiwan launched a national health insurance (NHI) program operated by a single buyer, the government. Medical reimbursement specialists and peer reviewers scrutinize all insurance claims. The diagnoses of diseases (including the five allergic diseases and neonatal jaundice) for variables and comorbidities in the NHIRD were based on the ICD-9-CM codes, which were strictly determined by related specialists and physicians according to the standard clinical diagnosis criteria. Therefore, the diagnoses and codes for the variables and comorbidities used in this study should be correct and reliable. Second, we analyzed the effects of several common allergic diseases. Third, the neonatal jaundice and nonneonatal jaundice cohorts were established by matching the patients according to sex and other sociodemographic factors to control for confounding factors. Fourth, we adjusted for numerous perinatal events (including fetal and newborn respiratory conditions, infections, preterm delivery and low birth weight, and other birth conditions) that may be associated with high levels of bilirubin and asthma. The Asian population is at a greater risk of neonatal jaundice than the populations in other geographic regions (32). Genetic variations such as the Gly71Arg mutation in the UGT1A1 gene are common in the Asian population, increasing the incidence of severe neonatal hyperbilirubinemia $(29,30,32)$.
Certain limitations should also be noted when interpreting our findings. In this study, we adjusted for most of the potential confounders, such as age, sex, and perinatal events (including fetal and newborn respiratory conditions, infections, preterm delivery and low birth weight, and other birth conditions), that may be associated with a high level of bilirubin and atopic diathesis. There might be some residual confounding factors, such as factors on which detailed clinical information is not included in the NHIRD (e.g., serum bilirubin level, serum IgE level, breastfeeding, and environmental exposures). In addition, estimates of the effect of the serum bilirubin level on subsequent atopic risk may have been biased. Furthermore, the study population consisted only of Chinese children; thus, our findings may not be generalizable to populations from other countries.

This study adds to the evidence of the association between neonatal jaundice and atopic diathesis. Children with neonatal jaundice are at an increased risk of several common allergic diseases; they were found to have the highest incidence rates for $\mathrm{AR}$, followed by asthma, AD, and urticaria. Further research is required to clarify the pathogenesis of these associations.

\section{METHODS}

\section{Data Sources}

The NHIRD, a population-based database, is maintained by the National Health Research Institutes and is derived from the claims data of the NHI program, a mandatory-enrollment, single-payment system created in 1995 and now covering over $99 \%$ of the Taiwanese population (http://www.nhi.gov.tw/english/index.aspx) (36). The children file (age $<18$ y) was derived from the NHIRD and is maintained by the National Health Research Institutes. The children file includes the claims data of $50 \%$ children randomly selected from the pediatric population enrolled during 1996-2008 (34-37). Diseases are coded on the basis of the International Classification of Diseases, Ninth Revision, Clinical Modification (ICD-9-CM). Because of personal electronic data privacy regulations, the information regarding the identification of insurants is encrypted before being sent to researchers. Hence, informed consent was not required for the current study. The study was approved by the Institutional Review Board of China Medical University Hospital (CMU-REC-101-012).

\section{Study Patients}

The cohort study included children with a new diagnosis of neonatal jaundice, identified according to ICD-9-CM code 774, from 2000 to 2007. The date of diagnosis of neonatal jaundice was set as the index date. Children aged $>1$ mo, those diagnosed with allergic disease before the index date, and those with missing information on sex or age were excluded from the study. For each child with neonatal jaundice, two children without neonatal jaundice from the same period were randomly selected and included in the nonneonatal jaundice cohort according to the aforementioned exclusion criteria. Patients with nonneonatal jaundice were frequency-matched with patients with neonatal jaundice according to sex, age, parental urbanization, parental occupation, and index date. A total of 27,693 patients with neonatal jaundice and 55,367 patients with nonneonatal jaundice were included in the current study.

\section{Outcome Measurement and Comorbidities}

Follow-up for each child in the neonatal jaundice cohort began on the first day of diagnosis of neonatal jaundice. The follow-up for each child in the nonneonatal jaundice cohort began on the matched index date. Both cohorts were followed up until the date of diagnosis of certain allergic diseases, death, withdrawal from the NHI program, or the end of 2008. To improve diagnostic reliability and to avoid the overestimation of prevalence, only children with at least one inpatient claim record 
or two ambulatory claims in any diagnosis field with respective ICD9-CM codes after the index date were designated as having a specific allergic disease. We identified the following atopic diseases by using ICD-9-CM codes: AC (372.05, 372.10, and 372.14), AR (477), asthma (493 and 494), AD (691), and urticaria (708.0 and 708.9). The prevalence rates of $\mathrm{AD}, \mathrm{AR}$, asthma, and urticaria in children aged 6-13 y in Taiwan in 2010 were $3.48,26.5,9.13$, and $6.73 \%$, respectively, according to the NHIRD (38). Recent surveys based on questionnaires designed by the International Study of Asthma and Allergies in Childhood show similar prevalence rates of allergic diseases in children $(39,40)$. In Northern Taiwan, the prevalence rates of $\mathrm{AD}, \mathrm{AR}$, and asthma in children aged $6-8$ y are $10.2,29.8$, and $12.2 \%$, respectively, and those in children aged $10-12$ y are $4.7,18.3$, and $9.6 \%$, respectively (39). In Central Taiwan, the prevalence rates of $\mathrm{AD}, \mathrm{AR}$, and asthma in children aged $6-15 y$ are $3.35,27.59$, and $6.99 \%$, respectively (40). No significant difference in the prevalence rate was observed between the study conducted using the NHIRD and studies conducted using International Study of Asthma and Allergies in Childhood questionnaires.

Neonatal respiratory conditions, perinatal infections, preterm delivery and low birth weight, birth conditions, the male sex, low socioeconomic status, and the urban environment were considered risk factors (Table 1) for atopy. A strong association exists between preterm delivery and asthma diagnosis. In addition, many very preterm newborns develop jaundice and are treated with phototherapy (11-13). We evaluated whether these factors influence the rates of development of five allergic diseases. When any influences were noted, the risk factors were considered confounding factors and were adjusted in subsequent study of the association between allergic diseases and neonatal jaundice. We incorporated data from inpatient and outpatient diagnosis files to ascertain comorbidities (identified according to ICD-9-CM codes), namely fetal and newborn respiratory conditions (769 and 770), infections (771), preterm delivery and low birth weight (764 and 765), other birth conditions (761-763 and 767-769), and glucose-6-phosphate dehydrogenase deficiency (282.2). Other birth conditions (classified according to ICD-9-CM codes) include fetus or newborn affected by maternal complications of pregnancy (761); fetus or newborn affected by complications pertaining to the placenta, cord, and membranes (762); fetus or newborn affected by other complications of labor and delivery (763); and birth trauma (764). The use of interventions for hyperbilirubinemia, including phototherapy and exchange transfusion, was investigated to determine the severity of neonatal jaundice.

\section{Statistical Analysis}

The demographic data of the neonatal jaundice and nonneonatal jaundice cohorts were compared using the $\chi^{2}$ test. Continuous data were expressed as the mean \pm SD and compared using the $t$-test. The Kaplan-Meier method and log-rank test were used to assess the differences in atopic event-free rates between the two cohorts. The incidence rates of atopic events were calculated for each cohort. A multivariate Cox proportional hazards model was used to calculate the HR and 95\% CI of developing an atopic event associated with neonatal jaundice. This model was simultaneously adjusted for age and sex. SAS software (Version 9.2 for windows; SAS Institute, Cary, NC) was used to perform all data analyses. The Kaplan-Meier plot was created using R software (Version 2.14.1, R Development Core Team, Vienna, Austria) to estimate the cumulative hazard. All statistical tests were set at a two-tailed significance level of 0.05 .

\section{ACKNOWLEDGENTS}

C.-C.W. and T.-C.S. conceptualized and designed the study, drafted the initial manuscript, and approved the final submitted manuscript. C.-L.L. and C.-H.K. conducted the initial analysis, reviewed and revised the manuscript, and approved the final submitted manuscript. C.-H.K. coordinated and supervised data collection, critically reviewed the manuscript, and approved the final submitted manuscript.

\section{STATEMENT OF FINANCIAL SUPPORT}

This study is supported in part by Taiwan Ministry of Health and Welfare Clinical Trial and Research Center of Excellence (MOHW104-TDU-B-212-113002); China Medical University Hospital, Academia Sinica Taiwan Biobank, Stroke Biosignature Project (BM104010092); NRPB Stroke Clinical Trial Consortium (MOST 103-2325-B-039 -006); Tseng-Lien Lin Foundation, Taichung, Taiwan;
Taiwan Brain Disease Foundation, Taipei, Taiwan; Katsuzo and Kiyo Aoshima Memorial Funds, Japan; and Health, and welfare surcharge of tobacco products, China Medical University Hospital Cancer Research Center of Excellence (MOHW104-TDU-B-212-124-002, Taiwan). The funders had no role in study design, data collection and analysis, decision to publish, or preparation of the manuscript. No additional external funding received for this study.

Disclosure: The authors have no financial relationships relevant to this article to disclose.

\section{REFERENCES}

1. Lauer BJ, Spector ND. Hyperbilirubinemia in the newborn. Pediatr Rev 2011;32:341-9.

2. Wennberg RP, Ahlfors CE, Bhutani VK, Johnson LH, Shapiro SM. Toward understanding kernicterus: a challenge to improve the management of jaundiced newborns. Pediatrics 2006:117:474-85.

3. Atkinson M, Budge H. Review of the NICE guidance on neonatal jaundice. Arch Dis Child Educ Pract Ed 2011;96:136-40.

4. Newman TB, Escobar GJ, Gonzales VM, Armstrong MA, Gardner MN, Folck BF. Frequency of neonatal bilirubin testing and hyperbilirubinemia in a large health maintenance organization. Pediatrics 1999;104 (5 Pt 2):1198-203.

5. Bélanger S, Lavoie JC, Chessex P. Influence of bilirubin on the antioxidant capacity of plasma in newborn infants. Biol Neonate 1997;71:233-8.

6. Minetti M, Mallozzi C, Di Stasi AM, Pietraforte D. Bilirubin is an effective antioxidant of peroxynitrite-mediated protein oxidation in human blood plasma. Arch Biochem Biophys 1998;352:165-74.

7. Jangi S, Otterbein L, Robson S. The molecular basis for the immunomodulatory activities of unconjugated bilirubin. Int J Biochem Cell Biol 2013;45:2843-51.

8. Haga Y, Tempero MA, Kay D, Zetterman RK. Intracellular accumulation of unconjugated bilirubin inhibits phytohemagglutin-induced proliferation and interleukin-2 production of human lymphocytes. Dig Dis Sci 1996;41:1468-74.

9. Romagnani S. The Th1/Th2 paradigm. Immunol Today 1997;18:263-6.

10. Romagnani S. The role of lymphocytes in allergic disease. J Allergy Clin Immunol 2000;105:399-408.

11. Aspberg S, Dahlquist G, Kahan T, Källén B. Is neonatal phototherapy associated with an increased risk for hospitalized childhood bronchial asthma? Pediatr Allergy Immunol 2007;18:313-9.

12. Aspberg S, Dahlquist G, Kahan T, Källén B. Confirmed association between neonatal phototherapy or neonatal icterus and risk of childhood asthma. Pediatr Allergy Immunol 2010;21(4 Pt 2):e733-9.

13. Ku MS, Sun HL, Sheu JN, Lee HS, Yang SF, Lue KH. Neonatal jaundice is a risk factor for childhood asthma: a retrospective cohort study. Pediatr Allergy Immunol 2012;23:623-8.

14. Huang LS, Bao YX, Xu ZL, et al. Neonatal Bilirubin Levels and Childhood Asthma in the US Collaborative Perinatal Project, 1959-1965. Am J Epidemiol 2013;178:1691-7.

15. Sun HL, Lue KH, Ku MS. Neonatal jaundice is a risk factor for childhood allergic rhinitis: a retrospective cohort study. Am J Rhinol Allergy 2013;27:192-6.

16. Källén B, Finnström O, Nygren KG, Otterblad Olausson P. Association between preterm birth and intrauterine growth retardation and child asthma. Eur Respir J 2013;41:671-6.

17. Crump C, Winkleby MA, Sundquist J, Sundquist K. Risk of asthma in young adults who were born preterm: a Swedish national cohort study. Pediatrics 2011;127:e913-20.

18. Kurt A, Aygun AD, Kurt AN, Godekmerdan A, Akarsu S, Yilmaz E. Use of phototherapy for neonatal hyperbilirubinemia affects cytokine production and lymphocyte subsets. Neonatology 2009;95:262-6.

19. Procianoy RS, Silveira RC, Fonseca LT, Heidemann LA, Neto EC. The influence of phototherapy on serum cytokine concentrations in newborn infants. Am J Perinatol 2010;27:375-9.

20. Aycicek A, Kocyigit A, Erel O, Senturk H. Phototherapy causes DNA damage in peripheral mononuclear leukocytes in term infants. J Pediatr (Rio J) 2008;84:141-6.

21. Tatli MM, Minnet C, Kocyigit A, Karadag A. Phototherapy increases DNA damage in lymphocytes of hyperbilirubinemic neonates. Mutat Res 2008;654:93-5. 


\section{Articles Wei et al.}

22. Maverakis E, Miyamura Y, Bowen MP, Correa G, Ono Y, Goodarzi H. Light, including ultraviolet. J Autoimmun 2010;34:J247-57.

23. Gloria-Bottini F, Bottini E. Is there a role of early neonatal events in susceptibility to allergy? Int J Biomed Sci 2010;6:8-12.

24. Ahlfors CE. Criteria for exchange transfusion in jaundiced newborns. Pediatrics 1994;93:488-94.

25. Johnson LH, Bhutani VK, Brown AK. System-based approach to management of neonatal jaundice and prevention of kernicterus. J Pediatr 2002;140:396-403.

26. La Rosa M, Romeo MG, Pratico G, Rizzo R, Mollica F. Serum IgE in newborns undergoing exchange transfusion. Ann Allergy 1986;57:259-62.

27. Gilliland FD, Li YF, Saxon A, Diaz-Sanchez D. Effect of glutathione-Stransferase M1 and P1 genotypes on xenobiotic enhancement of allergic responses: randomised, placebo-controlled crossover study. Lancet 2004;363:119-25.

28. Safronova OG, Vavilin VA, Lyapunova AA, et al. Relationship between glutathione S-transferase P1 polymorphism and bronchial asthma and atopic dermatitis. Bull Exp Biol Med 2003;136:73-5.

29. Abdel Ghany EA, Hussain NF, Botros SK. Glutathione S-transferase gene polymorphisms in neonatal hyperbilirubinemia. J Investig Med 2012;60:18-22.

30. Piacentini S, Polimanti R, Simonelli I, et al. Glutathione S-transferase polymorphisms, asthma susceptibility and confounding variables: a metaanalysis. Mol Biol Rep 2013;40:3299-313.

31. Raimondi F, Indrio F, Crivaro V, Araimo G, Capasso L, Paludetto R. Neonatal hyperbilirubinemia increases intestinal protein permeability and the prevalence of cow's milk protein intolerance. Acta Paediatr 2008;97:751-3.

32. Setia S, Villaveces A, Dhillon P, Mueller BA. Neonatal jaundice in Asian, white, and mixed-race infants. Arch Pediatr Adolesc Med 2002;156:276-9.

33. Davis K, Huang AT. Learning from Taiwan: experience with universal health insurance. Ann Intern Med 2008;148:313-4.

34. Wei CC, Tsai JD, Lin CL, Shen TC, Li TC, Chung CJ. Increased risk of idiopathic nephrotic syndrome in children with atopic dermatitis. Pediatr Nephrol 2014;29:2157-63.

35. Wei CC, Lin CL, Shen TC, Tsai JD, Chung CJ, Li TC. Increased incidence of juvenile-onset systemic lupus erythematosus among children with asthma. Pediatr Allergy Immunol 2014;25:374-9.

36. Wei CC, Lin CL, Kao CH, et al. Increased risk of Kawasaki disease in children with common allergic diseases. Ann Epidemiol 2014;24:340-3.

37. Tsai JD, Lin CL, Shen TC, Li TC, Wei CC. Increased subsequent risk of myasthenia gravis in children with allergic diseases. J Neuroimmunol 2014;276:202-6.

38. Kao CC, Huang JL, Ou LS, See LC. The prevalence, severity and seasonal variations of asthma, rhinitis and eczema in Taiwanese schoolchildren. Pediatr Allergy Immunol 2005;16:408-15.

39. Liao PF, Sun HL, Lu KH, Lue KH. Prevalence of childhood allergic diseases in central Taiwan over the past 15 years. Pediatr Neonatol 2009;50:18-25.

40. Tsai YJ, Lin CH, Fu LS, Fu YC, Lin MC, Jan SL. The association between Kawasaki disease and allergic diseases, from infancy to school age. Allergy Asthma Proc 2013;34:467-72. 